\title{
Correction to: The role of appetite-related hormones, adaptive thermogenesis, perceived hunger and stress in long-term weight- loss maintenance: a mixed-methods study
}

\author{
George Thom (1) - Stephan U. Dombrowski • Naomi Brosnahan · Yasmin Y. Algindan • M. Rosario Lopez-Gonzalez • \\ Giles Roditi $\cdot$ Michael E. J. Lean (1D) Dalia Malkova
}

Published online: 15 April 2021

(c) The Author(s), under exclusive licence to Springer Nature Limited 2021

Correction to: European Journal of Clinical Nutrition https://doi.org/10.1038/s41430-020-0568-9.

Since publication the authors noticed a missing data point in one figure (Fig. 4c) and a mistake in the interpretation of this figure, which made its way into the abstract, results and discussion sections of the paper.

Changes to the Abstract are as follows:

Results section

Original: 'Weight regain between 6 and 24 months $(6.1 \pm 6.3 \mathrm{~kg}, \quad P<0.05)$ was correlated positively with change in GLP-1 $(r=0.5, P=0.037)$ and negatively with GLP-1 at baseline $(r=-0.7, P=0.003)$ and after weight loss $(r=-0.7, P=0.005)$.'

Correct text: 'Weight regain between 6 and 24 months $(6.1 \pm 6.3 \mathrm{~kg}, P<0.05)$ was negatively correlated with GLP1 at baseline $(r=-0.7, P=0.003)$ and after weight loss ( $r=-0.7, P=0.005)$.'

Conclusion section

Original: 'Weight regain is more likely with lower fasting GLP-1 and greater reduction in GLP-1 after weight loss, but psychological aspects of eating behaviour appear as important in attenuating weight-loss maintenance.'

Correct text: 'Weight regain is more likely with lower fasting GLP-1at baseline and following weight-loss, but psychological aspects of eating behaviour appear as important in attenuating weight-loss maintenance.'

Changes to the Results are as follows:

Appetite-related hormones section

Original: 'Body weight regain from 6 to 24 months was positively correlated with changes in fasting GLP-1 concentration $(r=0.5, P=0.037) \ldots$ '
Correct text: 'Body weight regain from 6 to 24 months was weakly correlated with changes in fasting GLP-1 concentration $(r=0.5, P=0.051) \ldots$ '

Figure 2

Original description: 'Fig. 2 Individual changes in body weight $(\mathrm{kg}$ ) between (a) 0-6 months and (b) 6-24 months. Grey dashed lines indicate mean value (- - -). Body weight $(\mathrm{kg})$ at baseline, 6 months and 24 months for each participant.'

Correct text: 'Fig. 2 Body weight $(\mathrm{kg})$ at baseline, 6 months and 24 months for each participant. '

Changes to the Discussion are as follows:

Sentence starting on line 9

Original: 'This study suggests that individuals with higher fasting plasma GLP-1 concentrations and lower reduction in GLP-1 during diet-induced weight loss, and an ability to manage emotional difficulties without compensatory eating can be expected to be more successful at weight-loss maintenance.'

Correct text: 'This study suggests that individuals with higher fasting plasma GLP-1 concentrations and an ability to manage emotional difficulties without compensatory eating can be expected to be more successful at weight-loss maintenance.'

Final paragraph of discussion

Original: 'In conclusion, our findings imply that habitually higher fasting GLP-1 concentrations and lower reduction in GLP-1 during diet-induced weight loss form part of a favourable biological profile for long-term weightloss maintenance...'

Correct text: 'In conclusion, our findings imply that higher fasting GLP-1 concentrations form part of a favourable biological profile for long-term weight-loss maintenance...'

The original article has been corrected. 


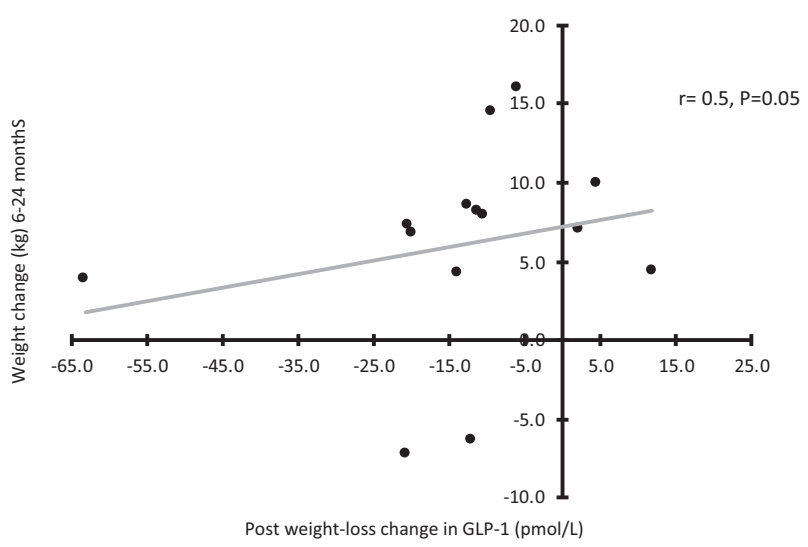

Fig. 4 Association between weight regain $(\mathrm{kg})$ from 6 to 24 months and (a) GLP-1 measured at baseline, (b) GLP-1 measured after weight loss, and (c) changein GLP-1 from baseline to 6 months. 